\title{
OBITUARIO DE LA CASA. LA VIVIENDA COMO EXPERIENCIA
}

\section{OBITUARY OF THE HOUSE. HOUSING AS EXPERIENCE}

\author{
J. Palacios Aguilar \\ Carrera de Arquitectura \\ Universidad de Lima -Perú \\ jpalacio@ulima.edu.pe
}

\begin{abstract}
RESUMEN
Obituario pretende reconocer el desuso y lo que ha dejado de existir en la casa (intrínseco o no), para describir luego todas esas cualidades inactivas y refundar la idea nuevamente. La estructura teórica-práctica del curso de Proyectos de Arquitectura VII (4to año carrera de 5) está centrada en el desarrollo de un proyecto de vivienda social colectiva. La idea del método es redescubrir esa casa que tenemos y a partir de ese momento re-diseñarla. Se les enseña que la práctica pedagógica (experiencia) del arquitecto se hace siempre desde lo ya construido, midiendo, dibujando, diseñando; a entender la vivienda desde la experiencia misma del cómo habitamos, haciéndonos partícipes del proceso mientras reformamos lo ya construido, lo ya habitado. Este acercamiento didáctico- pedagógico propone conducirlos a que sean ellos mismos los que produzcan sus propias herramientas de diseño, incentivarlos a manejar mediante la práctica misma del habitar -lo que corresponde o no al lugar.
\end{abstract}

Palabras Clave: habitar, medir, dibujar, proyectar

\begin{abstract}
Obituary aims to recognize disuse and has ceased to exist in the house (intrinsic or not) and then describe all these dormant qualities and refound the idea again. The structure (theoretical and practical) of the Project of Architectural Design VII (fourth year of five) focuses on the development of a social collective housing project. The idea of the method is to rediscover this house we already have and there after re-design it. They are taught that teaching practice (experience ) of the architect is always made from the already built, measuring, drawing, designing; understand the housing from the same experience of how to live, making us partakers of the process as we reform the already built, which already inhabited. This educational didactic approach proposes lead to they themselves produce their own design tools, encourage them to manage -through the same practice of dwelling -which fits with the place.
\end{abstract}

Keywords: inhabiting, measure, draw, design 


\section{OBITUARIO DE LA CASA}

Rescatando la mirada ausente

Los obituarios siempre son póstumos y en ello se pone de manifiesto esa voluntad de otorgarle un valor supremo a lo ausente; en nuestro caso intentamos asumir el riesgo de poner la casa en desahucio para con ello -casi inmediatamente- poner en vigencia el valor de su uso, revalorar lo intrínseco para recuperar luego el artefacto extrínseco, circunstancial.

Es un juego interesante sobre el valor del uso, los arquitectos vivimos proyectando desde lo existente (casa-ciudad) pero comprendiendo así mismo como cada espacio se funda desde su interrelación. Se plantea la idea del obituario desde la razón de los usos, funciones, medidas, espacios, valores cuantificables, interrelaciones entre ellos, grados de confort medidos desde las actividades, etc. y adecuarlos en esa mirada nueva. El espacio de la casa, el que habitamos necesita entenderse desde el uso que cada uno le otorga, mirarnos en ello es esencial para delimitar una posible solución.

Por ello la idea del "obituario de la casa" busca responder anticipadamente a las cualidades cuantitativas y cualitativas (obtener lo significativo, rescatar del ocio furtivo nuestras actividades), porque un obituario siempre reconstruye, reconstituye si bien está basado en un recuerdo, es momento oportuno para declarar lo constituido. Obituario siempre expone lo significativo del ser cuando desaparece, análogamente intentamos simular esa ausencia para reconstruir sobre lo existente.

El usuario es el protagonista, por ello "Obituario" se centra en ello y desde ahí incursionamos, la atención se fija desde rol del usuario. Las actividades que en ella se realizan y su vinculación con el entorno son un factor clave para comprender el rol del usuario sobre la vivienda, rol sobre su posición del contexto, y también frente a aquella inquietud teórica de resolver solo la unidad tipológica.

Enfocamos el aprendizaje de la vivienda desde la vida que en ella se desarrolla, repasar como vivirla es entenderla, redibujarla es comprenderla, medirla es apoderarse de ella; por eso tratar de encontrar los mecanismos que hacen posible una vida en ella es suficiente herramienta para proyectar. La vivienda es entendida como un conjunto de actividades en las que el espacio construido para ellas permite que éstas se den sin alterar ni romper las relaciones humanas concebidas, las no concebidas debieran poder constituirse en ese mismo marco. 


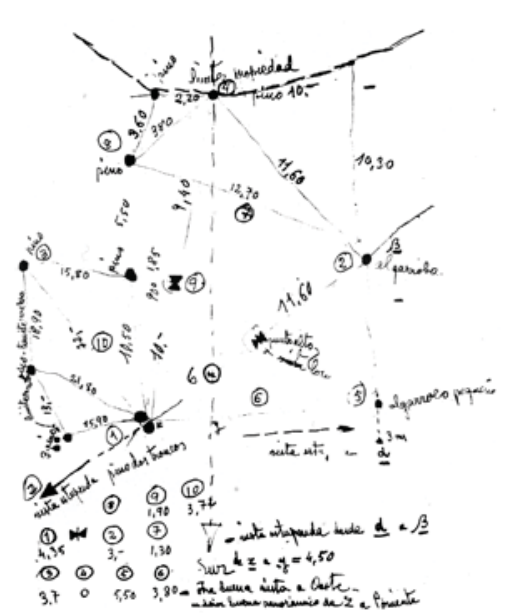

Figura 1_J.A. Coderch_Levantamiento árboles (1953-57) Casa Ugalde_Catalunya(1951)

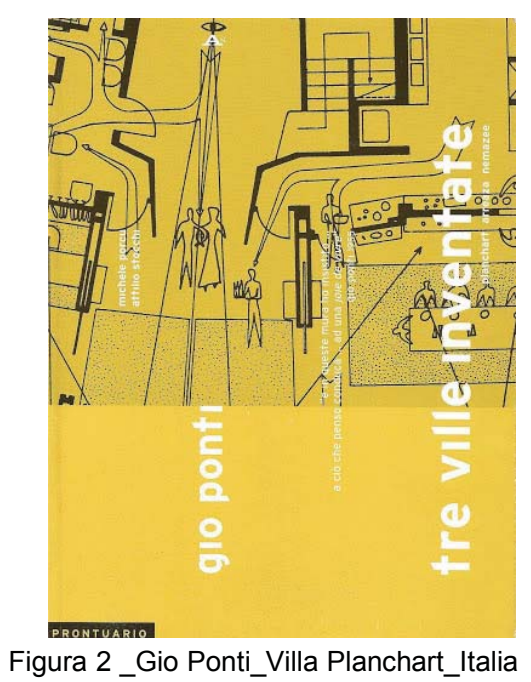

Figura 2_Gio Ponti_Villa Planchart_Italia

La idea de la cátedra es desprenderlos de lo habitual (diseñar solo con estrategias de diseño y no con herramientas proyectuales) para frenar un poco las ganas de ocupar el papel en blanco con bocetos si haber comprendido previamente el rol del habitante-entorno. No es el propósito fundamental aprender a diseñar una vivienda sino que entiendan mejor las suyas, sus "vínculos" (con la ciudad, con otras viviendas, equipamientos y más particularmente sobre los usos que comprende la suma de actividades). Porque pensamos que a partir dicha comprensión es que se estructura la disciplina del oficio, no tanto en el hacer sino en el comprender.

Entonces, ¿Porque nos centramos en el individuo? Porque nos basamos en sus desplazamientos más que en la casa como estructura? Porque el individuo soy yo, el que habita soy yo y "soy yo" son también todos ustedes, habitantes y ciudadanos. Sabemos que la vivienda compone gran parte del tejido urbano pero no lo organiza, lo organiza el espacio público - y su uso bien logrado lo reconstituye además- y el equipamiento. Hacer la ciudad es construirla desde varias miradas, pero si miramos siempre la vivienda como un ser y no como un individuo capaz de vincularse con su entorno vamos a construir lugares y no a constituir relaciones en ellas. Desde esa mirada es que se gesta el proyecto de vivienda, donde la vivienda jamás se desapega del individuo, porque lo entendemos como tal, donde la vivienda es la ciudad y la ciudad es la vivienda.

Trabajar con ellos desde su vivienda - apoyados con un ejercicio de referencias (ideológicas y arquitectónicas) para mover el enfoque- les llevará a comprender más sobre el lugar y el espacio de cada actividad. Nuestro rol es señalarles los mecanismos que hacen que una casa funcione mejor y/o porque no razón no podría funcionar. Nuestra actividad misma esta la calle, en las actividades que desarrollamos y compartimos en esos espacios de convivencia.

La casa debiera ser más un espacio que salvaguarda nuestros bienes y en la que descansamos y alimentamos, porque ahora es verdad que dichas 
actividades se puede dar en cualquier lugar, fuera de nuestra "casa" y no por eso le denominamos casa.

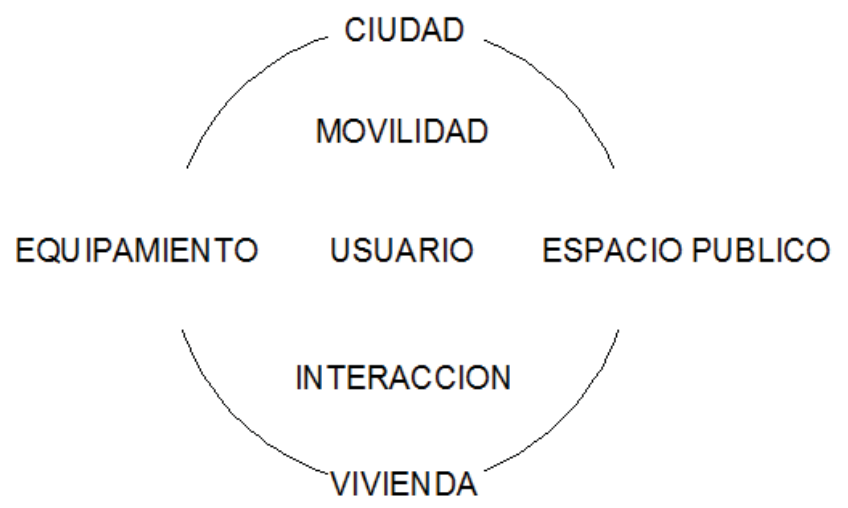

Figura 3_Diagrama que pretende construir el ámbito del curso_Proyecto Arquitectura VII 2014_I

El curso Proyecto de Arquitectura VII (4to año de carrera) se centra en esa sobreexposición de los hechos cotidianos (ciudad-casa). La sumilla del curso nos plantea trabajar sobre la vivienda colectiva y su relación con el entorno, pero para nosotros es más que comprender ambos sentidos. El curso está estructurado en 16 semanas, en todo momento ambas premisas (vivienda como tipología- vivienda en su relación con el entorno) se entrecruzan como un ADN donde el eje neurálgico que estructura ambas premisas es construido al mismo tiempo desde la experiencia y la teoría.

El curso se desarrolla sobre una zona urbana desatendida, elegida y estudiada previamente por la cátedra, haciendo énfasis en cómo plantear una alternativa de solución una vez identificada su problemática. Entre todo el taller (niveles IIIIX) - dispuesto en grupos verticales - deben de formular una mirada particular "una lectura propia del lugar de trabajo" que no consiste en contar postes de luz, reconocer las calles, marcar las área verdes, contar el mobiliario urbano, etc.

Esa mirada construida se convierte en una útil herramienta de diseño (identificar los tipos de usuarios, distancias y tiempos hacia equipamientos, como se da esa relevancia social del espacio en sus vidas, identificación de usuarios con lugares con actividades dinámicas y estáticas, tipos de movilidad, entornos de ocio, educación y servicios, etc.) cada uno de los grupos propone un plan maestro, que en diferentes etapas ( 3 semanas) es sometido a concurso interno (todos participan en la elección; docenes y alumnos) y finalmente el proyecto que se adecue a las necesidades del lugar es el trabajaran.

La idea de planificar y evaluar una parte de la ciudad para intervenirla es fundamental en su proceso de formación. . El plan ha determinado que tipo de estructura viaria, que servicios, que tipo de calles, de viviendas, como han definido la demarcación de lo público y lo privado. 
Entonces a partir de ese momento el Plan Maestro elegido se establece como punto de inicio para la siguiente etapa (8 semanas) cerrando esta etapa con una mesa redonda del equipo docente.

\section{Hacer público lo privado (Catalogando el orden)}

Hacer público lo privado tiene que ver con que nos revelen como se da la vida al interior de sus viviendas y como se desarrollan todo tipo de actividades en ella. Es ahora que les corresponde mostrarnos esa experiencia personal, vaciarse al interior, medir su ámbito; ya tienen la experiencia previa en donde midieron con herramientas construidas el ámbito territorial del encargo, ahora les corresponde construir herramientas también para medir su "espacio privado" y ver de qué manera los conectan con cada parte de esa prolongación del habitante que es la ciudad.

Lo primero es reconocerse en ellas, hacer un primer zoom a su vivienda, pero enfocado desde el dinamismo y la movilidad interior, no desde el cobijo (estructura física) identificando las actividades y programas de uso que en ella se desarrollan.

Analizar la relación funcional de su vivienda con respecto a los usuarios (familia) que la habitan es fundamental para proponer a futuro cualquier modificación -si las hubiere- en esta primera propuesta de reconocimiento.

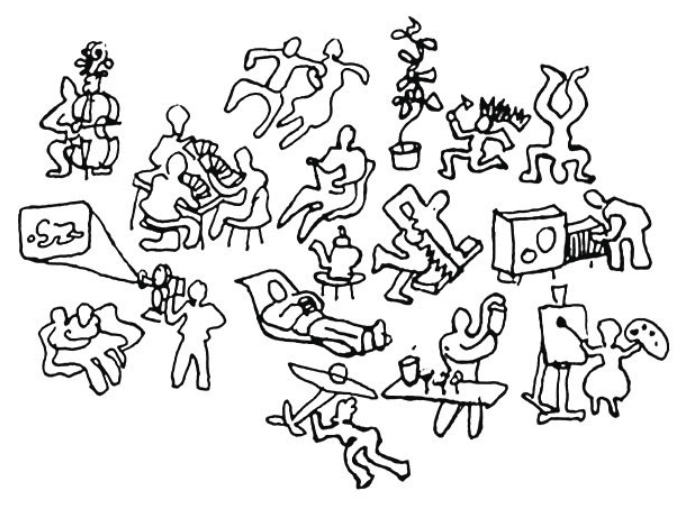

Figura 4_"What is a House"C.Eames_1944“
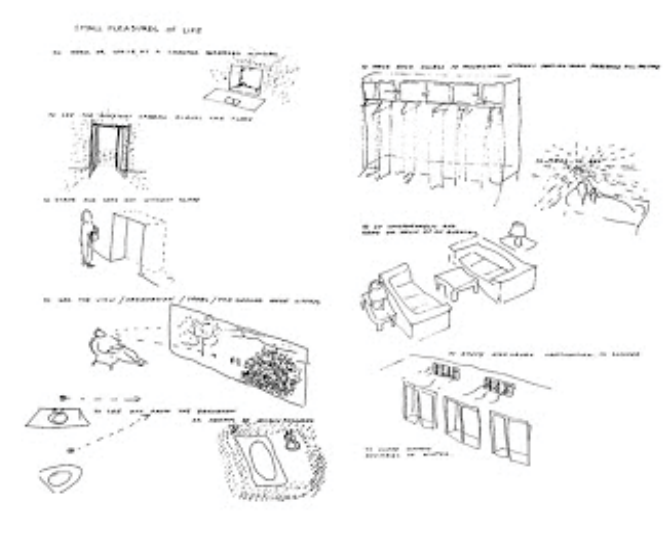

Iniciamos el proceso reconociéndose, de como viven, con quien habitan, cómo y con quien comparten su espacio privado, si viven solos o en familia, o no, si alojan gente particular (pensión), si reciben visitas de amigos esporádicamente, si trabajan en casa? si cenan o almuerzan solos o con sus padres a diario? si practican algún pasatiempo al interior, algún deporte, etc. etc. Estas preguntas que se plantean sobre la vivienda los llevan a cuestionarse -en el uso- si el lugar desde donde realizan las actividades antes mencionadas corresponde a su propia capacidad de albergarlos. El conjunto de todas estas acciones debería reconstruir lo cotidiano, construir las estelas dejadas por nuestros desplazamientos, debería también describir y descubrir los olores de la vida 
tanto al interior como al exterior y/o diferenciarlos al menos; para con ello poder catalogar un orden (espacio, tiempo, lugar). El medio para registrar todo aquello es mediante al observación y luego desde el dibujo; lápiz y papel en mano deben tomar notas y escribir buscando la sincronía (uso-arquitectura-función) que explique nuestro espacio de vida.

Mediante el reconocimiento de todas las actividades, incluso las no cotidianas es que comienza a aparecer una red de circuitos que permanecía invisible y/o poco atendida; el verdadero espacio de habitar se logra entender desde el uso. Para ello superponemos la malla de todas las actividades posibles sobre la tipología existente y con ello nos acercamos a nuestro grado de intimidad. Con ese mecanismo podemos saber; qué, cómo y para qué habríamos de modificar los ámbitos y las estancias o no (debería intervenirse y modificarse). Con ese diagrama de cosas tangibles e intangibles es que se vamos a producir un documento gráfico que nos sitúe en simultáneo con la realidad descubierta. Hemos hallado de esa manera la memoria activa que refleja ese amalgamiento entre lo que producimos como habitantes y lo que tenemos como lugar.
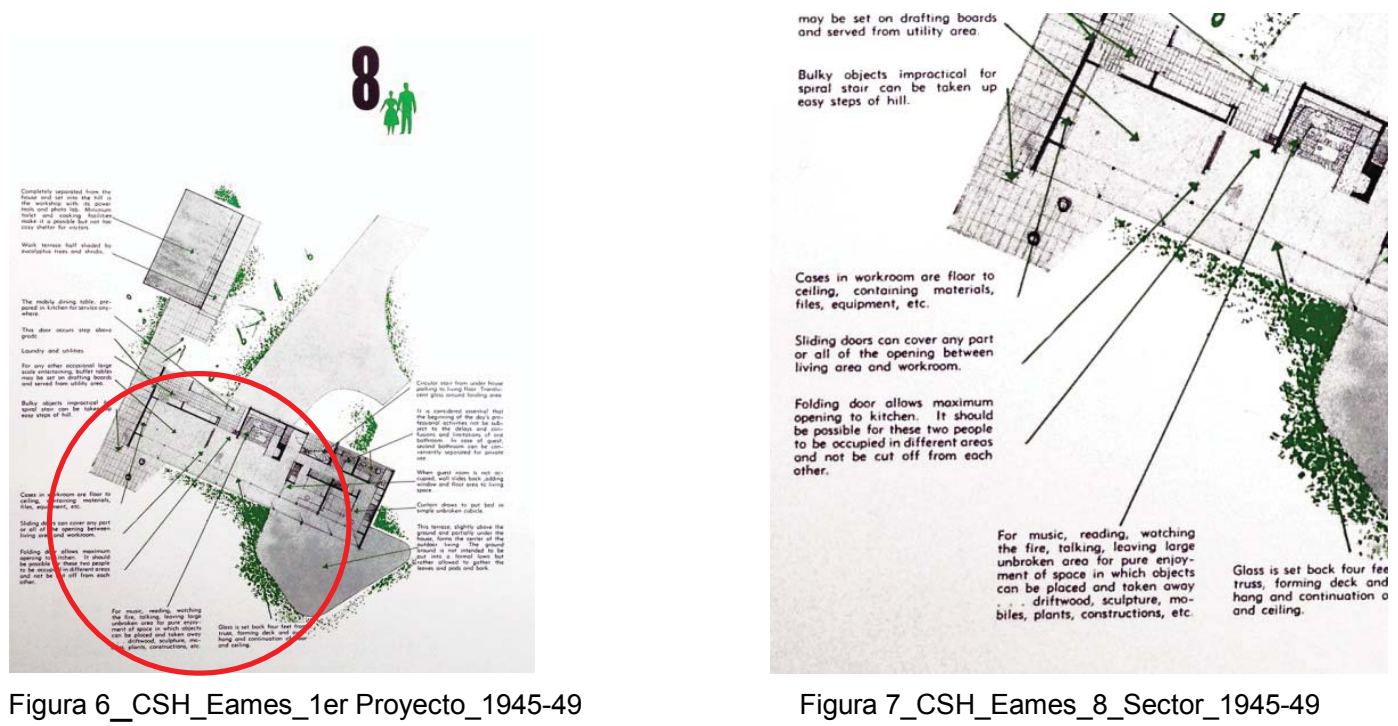

Figura 7_CSH_Eames_8_Sector_1945-49

Al igual que G.Ponti (Figura 2), los Eames (Figura 6-7) también le otorgan a la "actividad" toda su capacidad para desarrollarse sobre el espacio, (leer indicación de la figura 7 parte inferior del sector), por ello nos parece adecuado continuar trabajando sobre ese aspecto, ya que nos determinará el espacio necesario para cada movimiento. ¿Si en lo público sobre la ciudad reconocer y estudiar la actividad es fundamental porque no habría que analizarlo en la vivienda?

Desarrollando ese aspecto nos será más sencillo comprender los estados de la vivienda durante un periodo de tiempo determinado, en simultáneo, deberán además contabilizar todos los muebles y su capacidad de ocupación en cada 
estancia, buscar si es que se logran complementar o unir al dinamismo de las funciones establecidas, y si la estructura espacial que los alberga es parte fundamental sobre la función suscitada en ella.

Una vez que el material se convierte en evidencia proponemos una mesa redonda desde donde se le da valor a las relaciones que ayudan a que el habitar no se deteriore, donde las relaciones humanas al interior se favorecen, donde se tenga el confort deseable, que incorpore una adecuada iluminación y ventilación natural, y donde aparezca una posible y fresca mirada en el habitar. En el transcurso del desarrollo de sus propuestas realizamos visitas (02) a proyectos icónicos de vivienda social colectiva en Lima desde donde se explica la condición misma del programa y el tiempo como factor indispensable en la construcción social de la vivienda, con suerte podemos ingresar uno de los departamentos y conversar con los habitantes.

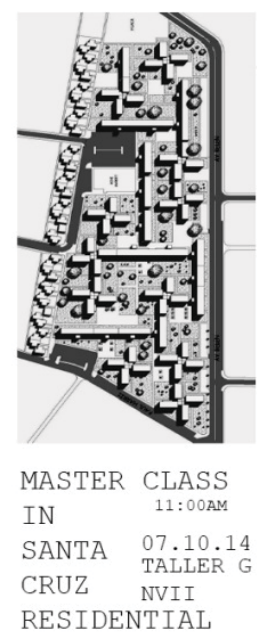

Figura 8_Poster de Visita N1_Taller G_2014-II

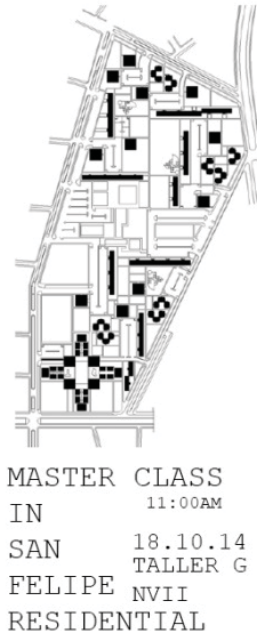

Figura 9_Poster de Visita N2_Taller G_2014-II

\section{Hacer público lo público y privado lo privado}

Con las conclusiones se comienza la segunda parte que corresponde a la transformación de su vivienda realizando los cambios necesarios que del análisis resultaron contraproducentes, donde el propio habitar les ha sugerido cambios, donde el espacio les va a determinar lo que es necesario para desarrollar todas las actividades catalogadas (desplazamientos y muebles).

Finalmente se presentan ambos documentos (antes y después), resaltando las relaciones físicas entre los habitantes, las relaciones entre el espacio físico y la arquitectura, la relación con los muebles, etc. Allí se cierra la etapa (ciudadvivienda) y comienza el desarrollo del proyecto sobre el Master Plan. Cuentan con todas las herramientas para proyectar, el sentido del orden y el uso lo han adquirido desde su propia experiencia.

No se trata de hacer edificios, ni dibujos de edificios que sean solamente la consecuencia de aplicarle áreas a un programa, desterramos proyectos 
reglamentarios (aplicación pura del Reglamento de Edificaciones) sino se trata de cuantificar y graficar una experiencia; en otras palabras cuantificar cada momento habitable. Se necesita que piensen cómo funcionan las cosas antes de proyectar. Antes de colocar el papel en blanco sobre la mesa deben ser conscientes de la responsabilidad que tienen, y sobre lo que se va a volcar en él, el levantamiento (de su vivienda) destruye la posibilidad del papel en blanco.

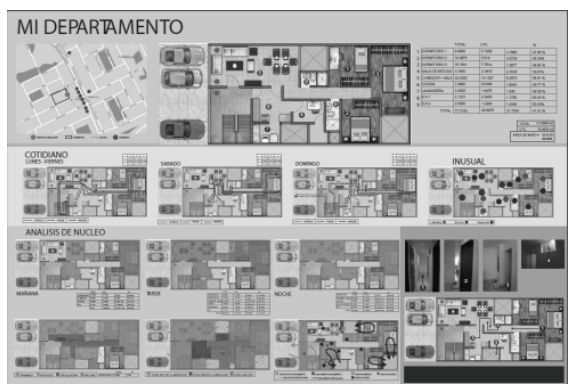

Figura 10_Levantamiento (áreas y desplazamientos) Alumna Almora_2014-II

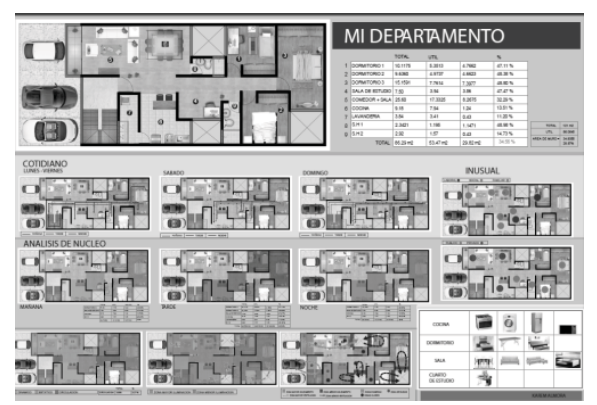

Figura 11 Reforma (áreas y desplazamientos) Alumna Almora_2014-II

Parte de nuestro trabajo es señalarles los lugares comunes, a veces, saber qué se necesita para proyectar es más certero que querer saber que proyectar.

Si tuviéramos que proyectar ahora un lugar, tendríamos más criterios que herramientas para proyectar, las herramientas casi vienen dadas. El lugar, la arquitectura que delimita todas estas actividades nacen desde la experiencia que es lo que buscamos en todo instante, no queremos hallar fórmulas, solo mecanismos para entender lo que proyectamos, no plantillas, y tampoco queremos inducir el programa con hábitos prefijados.
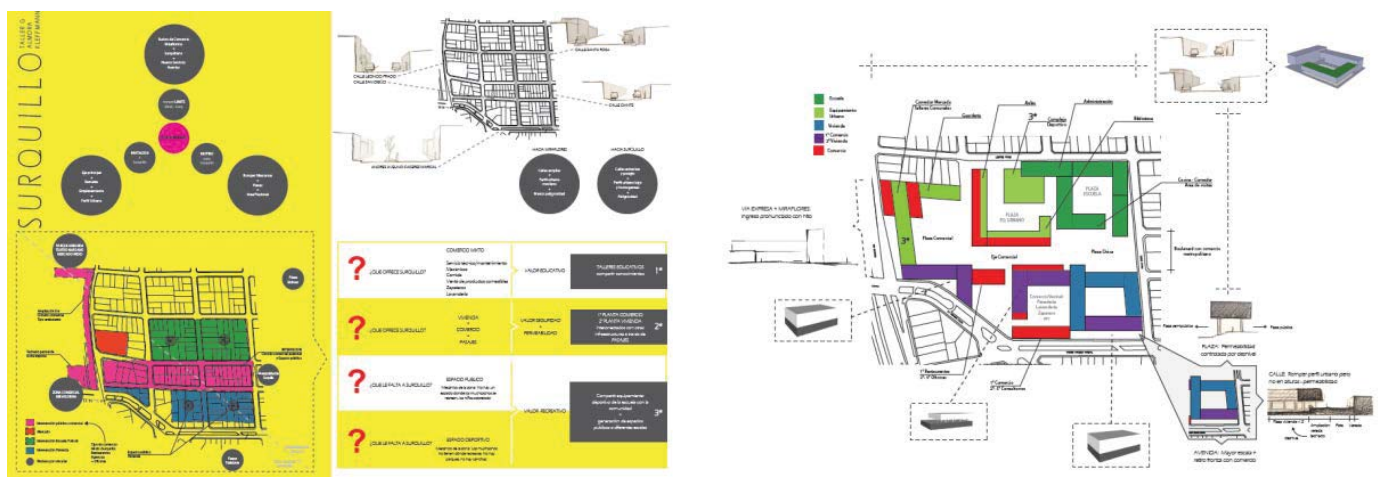

Figura 12-13_Master Plan: Análisis y Propuesta (Zona de equipamientos, viviendas, espacios públicos) _Alumnas Almora_Kleffmann_2014-II

La crítica del modelo tipológico viene dado por la evidencia de su experiencia revelada desde su propia condición del habitar. La condición más especial es que han descubierto cómo hacer para que el lugar donde viven y consumen funcione activamente para el individuo que lo habita. Este es un ejercicio fundamental antes de comenzar a hacer. 
Todo ello lo abordamos desde su experiencia, no nos interesa que produzcan infinidad de modelos tipológicos y que los repliquen por doquier hasta conseguir un volumen unitario con fachadas repetidas a los cuatro vientos y que luego fueran representarlos en una maqueta a gran escala con efectos gravitatorios incluso. Desterramos desde el inicio los dogmas y las fórmulas para resolver el problema de la cada pieza en la ciudad y por ende de la vivienda, no es lo que pretendemos, todo lo contrario, deseamos siempre poder fabricar cualquier tipo de herramientas. Al entregarles herramientas para pensar, estamos buscando primeramente que se identifiquen como ciudadanos, no solo que sean o aspiren a ser buenos arquitectos, parte de nuestra labor es ayudarles a construirse tomando ese sedimento cultural, social y arquitectónico que nos conforma como habitantes, ya que toda esta experiencia les servirá para reflexionar en cada actuar de su vida.

Aparece siempre nuestro esfuerzo por generar conciencia de su actitud frente a la ciudad, que sean conscientes de su experiencia como habitantes, ¿porque casi siempre asumimos que no tienen experiencia? si llevan toda su vida habitando. Lo que se pretende es otorgarles herramientas para que vayan extrayendo poco a poco esa experiencia que llevan consigo y puedan aplicarla al momento de reflexionar sobre la condición de la vivienda que les tocará desarrollar. En el enfoque predeterminado de la vivienda no se niegan las referencias arquitectónicas, solo se retarda su utilización como dispositivo para generar nuevas herramientas, primero necesitamos que produzcan las suyas (desde la actividad) y luego que vayan sumando decisiones proyectuales.

El ámbito de la vivienda en éste curso está enfocado para gente con pocos recursos porque es desde allí donde debemos de trabajar y proponer una optimización del recurso espacial y de sus relaciones. Trabajando eficazmente las áreas del programa podríamos crear regulaciones en la tipología y así proponer un espacio adecuado para la vivienda y adaptarlos a los modos de vida de hoy sin alterar los vínculos sociales y de convivencia entre los usuarios. De esa manera podríamos obtener como resultado una diversidad tipológica con una buena capacidad espacial y con posibilidades en abiertas desde el manejo en la estrategia proyectual, por lo menos al inicio.

El encargo (viene fijado en el Master Plan) debe desarrollarse en un área de $1000 \mathrm{~m} 2$, donde la densidad es de 1000hab/Ha, deben dejar el 50\% del área sin edificar y las tipologías a desarrollar tienen que establecer la relación con el usuario previamente identificados en el análisis. La altura del edificio bien dado igualmente por el estudio del Master Plan, si creen conveniente modificar algo en del plan, este deberá estar debidamente justificado. Las medidas de la tipología sugeridas son de 60, 90,120m2 de medida como máximo, pudiendo desarrollarse en flat, dúplex y triplex si ello les va a ayudar a regular la densidad y la diversidad en la sección. Debiendo incorporar de manera clara los criterios arquitectónicos explorados en todas las etapas, abordando las condiciones climáticas, de confort, de uso, estructurales, etc. La cantidad de vehículos se estima a 1 vehículo/ unidad de vivienda. 

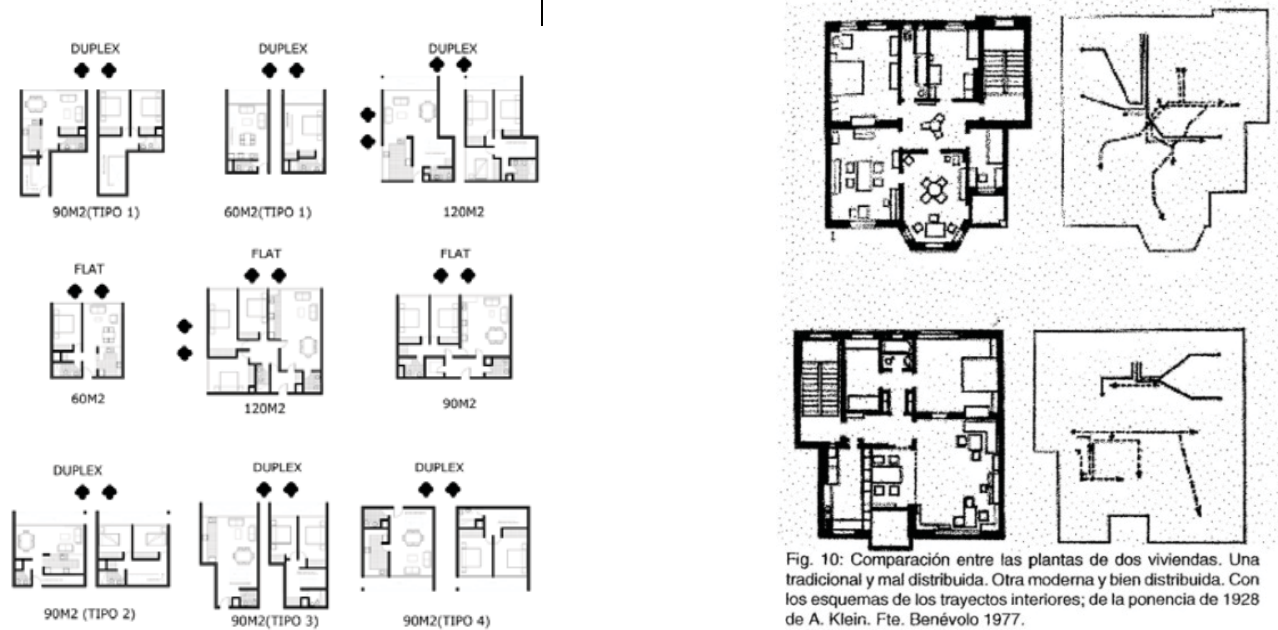

Figura 14_Tipologías diversas Flat y Duplex_Alumno Rivero_2014-II

Figura 15_Referencia_Alexander Klein_estudio sobre una misma tipologia_1928

Los proyectos son desarrollados en distintas escalas, el Master Plan 1:500 (etapa grupal), propuesta de bloque de viviendas (individual) 1:200; y una entrega previa 1:100 (planos y maqueta) y la entrega final es una sección 1:50 con planos completos, maqueta y detalles de fachadas. La entrega es acompañada de un panel A-1 donde se detalla todo el proceso. La penúltima semana se entregan los planos y la última semana la maqueta. Deberán traer la propuesta 1:200 inicial y se presenta junto con el Master Plan.

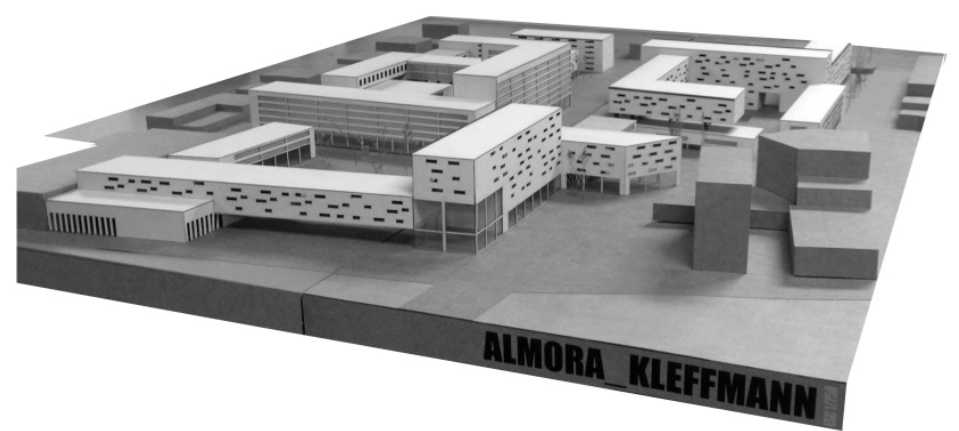

Figura 15_Master Plan_Alumnas Almora_Kleffmann_2014-II 


\section{Conclusiones}

Una vez comprendido el rol del usuario desde el colectivo ciudadano (afrontar necesidades urbanas colectivas), las dinámicas de la ciudad, la movilidad y los desplazamientos, se ha aprendido que la ciudad nunca deja de ser dinámica y es el usuario quien la dibuja desde su habitar, trabajar con las huellas de esos trazos es fundamental para construir herramientas. Las herramientas de análisis obtenidas en la lectura de la ciudad son fundamentales para construir situaciones de relación e interacción entre los ciudadanos.

EL organizar e intervenir la ciudad desde el entendimiento del espacio público, equipamiento urbano y vivienda nos ayuda a entender que la ciudad es un todo y debemos de pensarla como un solo ámbito siempre. La vivienda no es solo un edificio sino su relación con el entorno edificado y como se integra sin destruir las relaciones humanas que allí se dan, entenderlo desde su formación como futuros arquitectos ayudará a su contribución como ciudadanos a-arquitectos.

El dibujo y redibujo de las actividades en su vivienda los acerca al oficio del arquitecto en dos sentidos: levantar información para construir y reconstituir, y también comprender el dinamismo activo del usuario que predetermina la lógica del proyecto.

Las tipologías resultantes han sido resueltas desde el entendimiento más que del propio diseño. Son el resultado de comprender el uso del espacio en función de su propia individualidad.

Mediante el estudio de sus propuestas han comprendido que las necesidades y las cosas que nos rodean habitualmente son fundamentales y que reflejan una presencia constante en la ciudad y en menor escala en nuestra vivienda. Los bienes muebles e inmuebles pertenecen al imaginario de nuestra vida cotidiana y tenemos que ser muy conscientes del espacio que les corresponde y del espacio que ocupan en la casa y en la ciudad.

Prepararlos desde la condición cívica que debe de ejercer el ciudadano es parte de nuestra labor como educadores, tenemos el deber de proponerles que deben de estudiar sus hábitos, cambios y formas de vincularse, prestarles atención sin olvidar su condición porque la ciudad la hacemos todos y cada uno de nosotros. 